\title{
Assessment of Readiness and Usability of Information Systems Use
}

\author{
Dwi Yuniarto ${ }^{1}$, A'ang Subiyakto ${ }^{2}$, Aedah Binti Abd. Rohman ${ }^{3}$, Reny Rian Marliana ${ }^{4}$ \\ ${ }^{1,4}$ Informatics Engineering, STMIK Sumedang, Indonesia \\ ${ }^{2}$ Informatioan System Department, UIN Syarief Hidayatullah Jakarta, Indonesia \\ ${ }^{3}$ Department of Information Communication Technology, Asia e University, Malaysia \\ 11duart0@stmik-sumedang.ac.id, ${ }^{2}$ aang_subiyakto@uinjkt.ac.id, ${ }^{3}$ aedah.abdrahman@aeu.edu.my, ${ }^{4}$ reny@stmik-sumedang.ac.id
}

\begin{abstract}
The assessment of the use of information systems has been carried out by many researchers. This research was conducted in Private Universities in Indonesia, which currently involve many information systems in many ways, especially those related to the management of Higher Education, by measuring the readiness and usability of the use of information systems with models that I build from the integration of two models. The results of the measurement of this study were obtained from the distribution of questionnaires, there were $47 \%$ of respondents who filled $61-80 \%$ of the level of IS usage and $68 \%$ of respondents stated their readiness in the level of readiness to use IS. The stage consists of evaluating reflective measurement models and structural model assessments. Evaluating reflective measurement in evaluating internal consistency reliability using Composite Reliability, Reliability Indicator, Convergent Validity, and Discriminant Validity, finally concluded that the use of the Readiness and Usability integration model can be forwarded to a more complex research stage and can use the questionnaire.
\end{abstract}

Keywords- assessment, readiness, usability, use of information systems

\section{INTRODUCTION}

As information systems have become widely utilized, the assessment of the information system has likewise become an important research topic [1]. The Information system has been narrowly defined in terms of databases [2]. This definition focuses on data requirements and the mechanism to store, organize, process, and analyze data [315]. An alternative definition takes on a broad perspective to encompass all components of the system, such as data, software and hardware, people, methods, and procedures [16]. The use of information systems in universities has become a necessity $[17,18]$, some research on information systems in universities has increased along with the increasing need for information systems in universities [19-22].

The internationalization of economies, globalization, the rapid advance of the new technology, changes in production structures, business reorganization and so forth all place increasing pressure on the national statistical systems [23]. Research in science and engineering often involves using controllable and/or easy-to-measure variables (factors) to explain, regulate, or predict the behavior of other variables (responses) [24]. According to past literature study, some researchers used PLS [12, 2533] method for the analysis. Structural Equation Modeling [34] is one of the current methods used to cover the existing weaknesses of the regression method. The method experts SEM research grouping into two approaches [35]. The first approach is called as Covariance Based SEM (CBSEM) and the other approach is Variance Based SEM or better known as Partial Least Squares (PLS) [36].

In this study, statistical analysis was used to test the questionnaire on the use of information systems derived from the indicators of each variable made from the results of integrating the model of readiness and usabiility. The aim is to statistically assess the results of questionnaire analysis. The findings of this study can later provide input for researchers in terms of testing questionnaires and revising questionnaires, especially in the use of information systems. The research questions used in this study are:

Q1: What is the effect of readiness and usability on the use of information systems?

Q2: Are the results of the assessment of the use of information systems in terms of readiness and usability changing the indicators and variables that exist?

This research was carried out sequentially through four stages of research. At the first stage, the researcher explained the background of the results of the study. The explanation of the research method is in the second stage which explains the stages in the research procedure that is carried out and an explanation of the model used in the measurement of the use of information systems. The third stage of this study presents the results and discussions that are the answers to the problems as stated in the first stage. Finally, from this stage of the research, a conclusion can be drawn which can illustrate the desired results of this study.

\section{METHOD}

The scope of this study consists of preliminary studies (ie, literature review, model development, and instrument development studies), research programs, model development, research models, instrument development, research instruments, data collection, data analysis, analysis results, interpretation, interpretation result, report writing and result analysis, as stated in Figure 1. The input 
from this study is the model that has been produced by integrating the readiness and usability model (Figure 2) which has produced the questionnaire that will be evaluated.

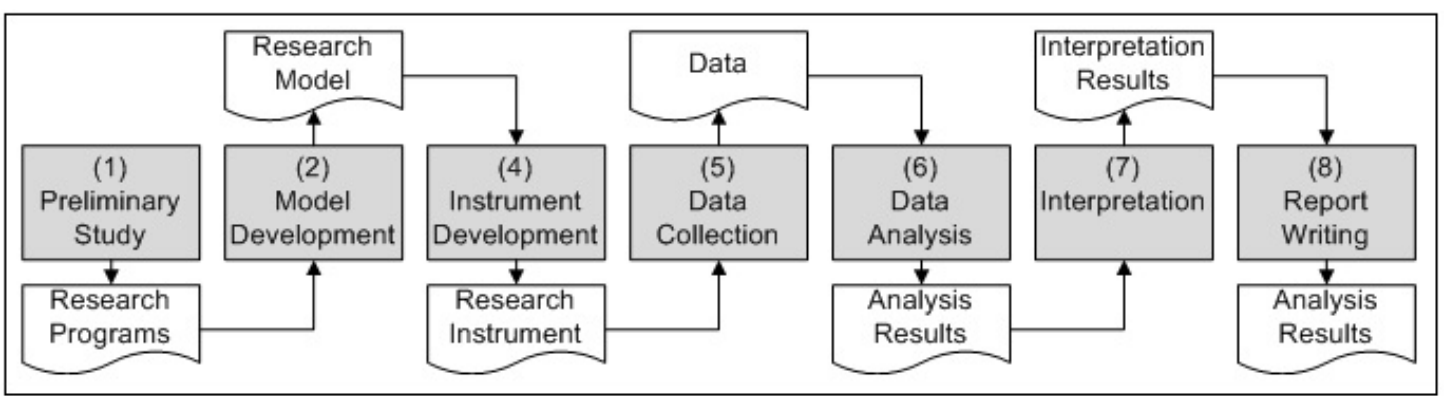

Figure 1. The research procedure [12]

This study developed an information system use model (Figure 2) by adopting Technology Readiness [37] and Usability [38]. The ten variables of the developed model are OPT (Optimism), INV (Innovation), DCF (Discomfort), ISC (Insecurity), LRN (Learnability), EFC (Efficiency), MMR (Memorability), RLB (Reliability), STF (Satisfaction), and SYU (System Usability), From the model that has been built, a questionnaire is generated which is a derivative of the indicators of the variables contained in the model (Figure 2) [2] (Table I and II).

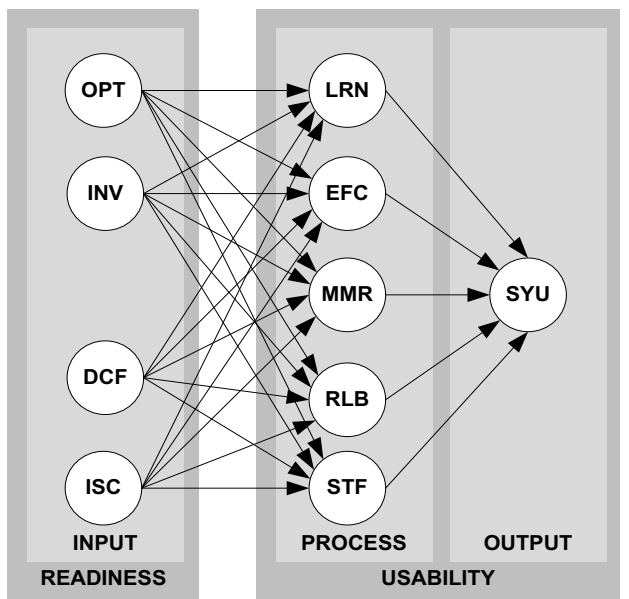

Figure 2 .The Developed Use Information System [2]

The researcher distributed 60 copies of questionnaires, which were distributed based on the experience of the respondent's profile. The distribution of questionnaires is done through the Google Form. The researchers processed the collected data using SmartPLS 2.0. Regarding the amount of data, the PLSSEM method is then used in the analysis phase by using SmartPLS 2.0 to perform reliability indicators, internal consistency reliability, convergent validity, and discriminant validity assessment.

Table 1. List of the questions

\begin{tabular}{cl}
\hline Code & \multicolumn{1}{c}{ Questionnaire } \\
\hline OPT1 & The System is free from constraints, difficulties, and troubles \\
& {$[3]$} \\
OPT2 & The System can be connected easily with other systems [3] \\
OPT3 & The System operates within the minimal resources [3] \\
OPT4 & The System operates within the maximal output [3] \\
OPT5 & The System is able to operate efficiently and effectively [3]
\end{tabular}

\begin{tabular}{|c|c|}
\hline Code & Questionnaire \\
\hline INV1 & A System is a problem-solving tool for users [3] \\
\hline INV2 & $\begin{array}{l}\text { The System helps users to be free from the controls/influences } \\
\text { [3] }\end{array}$ \\
\hline INV3 & $\begin{array}{l}\text { The System supports users for achieving goals in a difficult } \\
\text { situation or problem [3] }\end{array}$ \\
\hline INV4 & The System encourages users to achieve goals [3] \\
\hline INV5 & $\begin{array}{l}\text { The System supports users to be more successful than their } \\
\text { competitors [3] }\end{array}$ \\
\hline DCF1 & The System confuses users in its operation [3] \\
\hline DCF2 & The System cannot be operated easily [3] \\
\hline DCF3 & The System cannot be operated freely [3] \\
\hline DCF4 & The System is operated without a full support operation [3] \\
\hline DCF5 & The System is inappropriate to its development planning [3] \\
\hline ISC1 & $\begin{array}{l}\text { The System is unsuccessful be operated appropriated to its } \\
\text { development planning [3] }\end{array}$ \\
\hline ISC2 & $\begin{array}{l}\text { The System is in a situation that could cause harm or danger } \\
\text { [3] }\end{array}$ \\
\hline ISC3 & The System makes users become less in interactions [3] \\
\hline ISC4 & $\begin{array}{l}\text { The System makes users be unfocused with their importance } \\
\text { [3] }\end{array}$ \\
\hline ISC5 & The system is dubious to use [3] \\
\hline LRN1 & The system is easy to use [2] \\
\hline LRN2 & The system is very simple [2] \\
\hline EFC1 & The system gets the job done effectively [2] \\
\hline $\mathrm{EFC} 2$ & The System quickly completes the job [2] \\
\hline EFC3 & The system gets the job done efficiently [2] \\
\hline MMR 1 & The information in this SI is easy to understand [2] \\
\hline MMR2 & Their commands are aligned to specific functions [2] \\
\hline MMR3 & There hierarchical of the interface is simple to understand [2] \\
\hline RLB1 & The system is always available to operate when needed [2] \\
\hline RLB2 & $\begin{array}{l}\text { The System is protected from physical access from non- } \\
\text { authoritative rights [2] }\end{array}$ \\
\hline RLB3 & The system is easy to maintenance [2] \\
\hline RLB4 & The system processing is complete, accurate, and timely [2] \\
\hline STF1 & In this SI, the information provided is very clear [2] \\
\hline STF2 & In this SI there is ease in finding the information needed [2] \\
\hline STF3 & Their navigation in the interface is satisfactory [2] \\
\hline STF4 & The input method is appropriate [2] \\
\hline SYU1 & The organization of information on the screens was clear [2] \\
\hline SYU2 & The interface of this system was pleasant [2] \\
\hline SYU3 & I liked using the interface of this system [2] \\
\hline SYU4 & This system has all the functions and I expect it to have [2] \\
\hline SYU5 & Overall, I am satisfied with this system [2] \\
\hline
\end{tabular}

Table 2. List of the variables and indicators

\begin{tabular}{|c|c|c|}
\hline Variables & Indicators & References \\
\hline Optimism & $\begin{array}{l}\text { Easiness, connectivity, efficiency, } \\
\text { effectiveness, productivity. }\end{array}$ & {$[37,39-43]$} \\
\hline Innovation & $\begin{array}{c}\text { Problem solving, independence, } \\
\text { challenge, stimulation, competitiveness }\end{array}$ & $\begin{array}{c}{[15,37,43-} \\
45]\end{array}$ \\
\hline Discomfort & $\begin{array}{l}\text { Complexity, difficulty, dependence, } \\
\text { lack of support, inappropriateness }\end{array}$ & {$[37,39-43]$} \\
\hline Insecurity & $\begin{array}{l}\text { Failure, threat, reducing interaction, } \\
\text { distraction, incredulity }\end{array}$ & $\begin{array}{c}{[37,39-42,} \\
46]\end{array}$ \\
\hline Learnability & Ease of use, simplicity & {$[38]$} \\
\hline Efficiency & Effectively, quickly, efficiency & [38] \\
\hline
\end{tabular}

Assessment of Readiness and Usability of Information Systems Use

(Dwi Yuniarto, A'ang Subiyakto, Aedah Binti Abd. Rohman, Reny Rian Marliana) 
JOIN | Volume 4 No. 1 Juni 2019 : 1-8

\begin{tabular}{|c|c|c|}
\hline Variables & Indicators & References \\
\hline Memorability & $\begin{array}{l}\text { Understanding, functionality, } \\
\text { convenience }\end{array}$ & [38] \\
\hline Reliability & $\begin{array}{c}\text { Availability, protectivity, maintenance, } \\
\text { accuracy }\end{array}$ & $\begin{array}{l}{[37,38,47-} \\
49]\end{array}$ \\
\hline Satisfaction & $\begin{array}{l}\text { Clearly, easily, satisfaction, } \\
\text { appropriately }\end{array}$ & [38] \\
\hline $\begin{array}{l}\text { System } \\
\text { Usability }\end{array}$ & $\begin{array}{l}\text { Obviously, pleasantly, likely, } \\
\text { expectation, excitement }\end{array}$ & [38] \\
\hline
\end{tabular}

\section{RESULTS AND DISCUSSION}

\section{A. Demographics Information}

Table III presents the characteristics of the respondents, i.e., education, position, experience and skill level in using IS. From the results of data collection, it can be seen in Table 4 regarding the characteristics of respondents in terms of readiness and usability in the use of information systems. The above results can provide a recommendation for researchers in terms of data consistency between data collected by the expectations of the researchers. There were $47 \%$ of respondents who filled $61-80 \%$ of the level of IS usage and $68 \%$ of respondents stated their readiness in the level of readiness to use IS.

Table 3. Respondents profiles

\begin{tabular}{ccc}
\hline Measures & Items & $\mathbf{\%}$ \\
\hline Education & High School & 3 \\
& Diploma & 0 \\
& Bachelor & 12 \\
& Master & 75 \\
& Doctor & 10 \\
\hline Position & Top Manager & 22 \\
& Business Unit Manager & 20 \\
& Project Manager & 47 \\
& Project Team Member & 12 \\
\hline Experience & $<2$ years & 18 \\
& 2-5 years & 35 \\
& 5-10 years & 18 \\
& $>10$ years & 28 \\
\hline Skill & Very unskilled & 0 \\
& Unskilled & 0 \\
& Less skilled & 23 \\
& Skilled & 58 \\
& Very skilled & 18 \\
\hline
\end{tabular}

Table 4. Readiness and usability profiles

\begin{tabular}{ccc}
\hline Measures & Items & $\mathbf{\%}$ \\
\hline Strategic Plan & Exist & 82 \\
& No & 5 \\
& Unknown & 13 \\
\hline Level of Readiness to use & Very unprepared & 0 \\
IS & Not ready & 0 \\
& Less ready & 15 \\
& Ready & 68 \\
& Very ready & 17 \\
\hline Level of IS Usage & $<20 \%$ & 5 \\
& $21-40 \%$ & 7 \\
& $41-60 \%$ & 27 \\
\hline
\end{tabular}

\begin{tabular}{ccc}
\hline Measures & Items & $\mathbf{\%}$ \\
\hline & $61-80 \%$ & 47 \\
& $81-100 \%$ & 15 \\
\hline Factors that influence the & Cost availability & 47 \\
readiness of IS Usage & HR availability & 32 \\
(Technical) & Technology availability & 12 \\
& Data availability & 7 \\
& Method availability & 3 \\
\hline Factors that influence the & Cost availability & 33 \\
readiness of IS Usage & HR availability & 25 \\
(Managerial) & Technology availability & 12 \\
& Data availability & 12 \\
& Method availability & 18 \\
\hline Factors that influence the & The current SI Concert & 17 \\
readiness of IS Usage & Culture and work systems & 40 \\
(Institutional) & Support and coordination & 20 \\
& Staff support and commitment & 10 \\
& Leadership support and commitment & 13 \\
\hline IS Advantage & Technical handling of tasks & 17 \\
& Operational services & 22 \\
& Managerial business & 8 \\
& Institution Strategic & 53 \\
\hline Readiness Factors Affect & Not very influential & 2 \\
the IS Usage & No effect & 2 \\
& Less influential & 2 \\
& Take effect & 50 \\
& Very influential & 45 \\
\hline & &
\end{tabular}

From the results of data collection, it can be seen in Table 4 regarding the characteristics of respondents in terms of readiness and usability in the use of information systems. The above results can provide a recommendation for researchers in terms of data consistency between data collected by the expectations of the researchers. There were $47 \%$ of respondents who filled $61-80 \%$ of the level of IS usage and $68 \%$ of respondents stated their readiness in the level of readiness to use IS.

B. The Statistical Analysis Result

At the statistical analysis result stage, there are several stages to process the questionnaire. The stage consists of evaluating reflective measurement models and structural model assessment. Evaluating reflective measurement are evaluating internal consistency reliability using Composite Reliability (Table IV and Tabel VI), Indicator Reliability (Tabel V), Convergent Validity (Table VII), Discriminant Validity (Tabel VIII).

The Structural Model Assessment is a step to determining whether or not the hypothesis is based on the research model (Table IX), assessing $\mathrm{R}^{2}$ values of the endogenous latent variable(s) in the path model (Table X) and the last step is assessing an exogenous construct's contribution to an endogenous latent variable's (Table XI). 


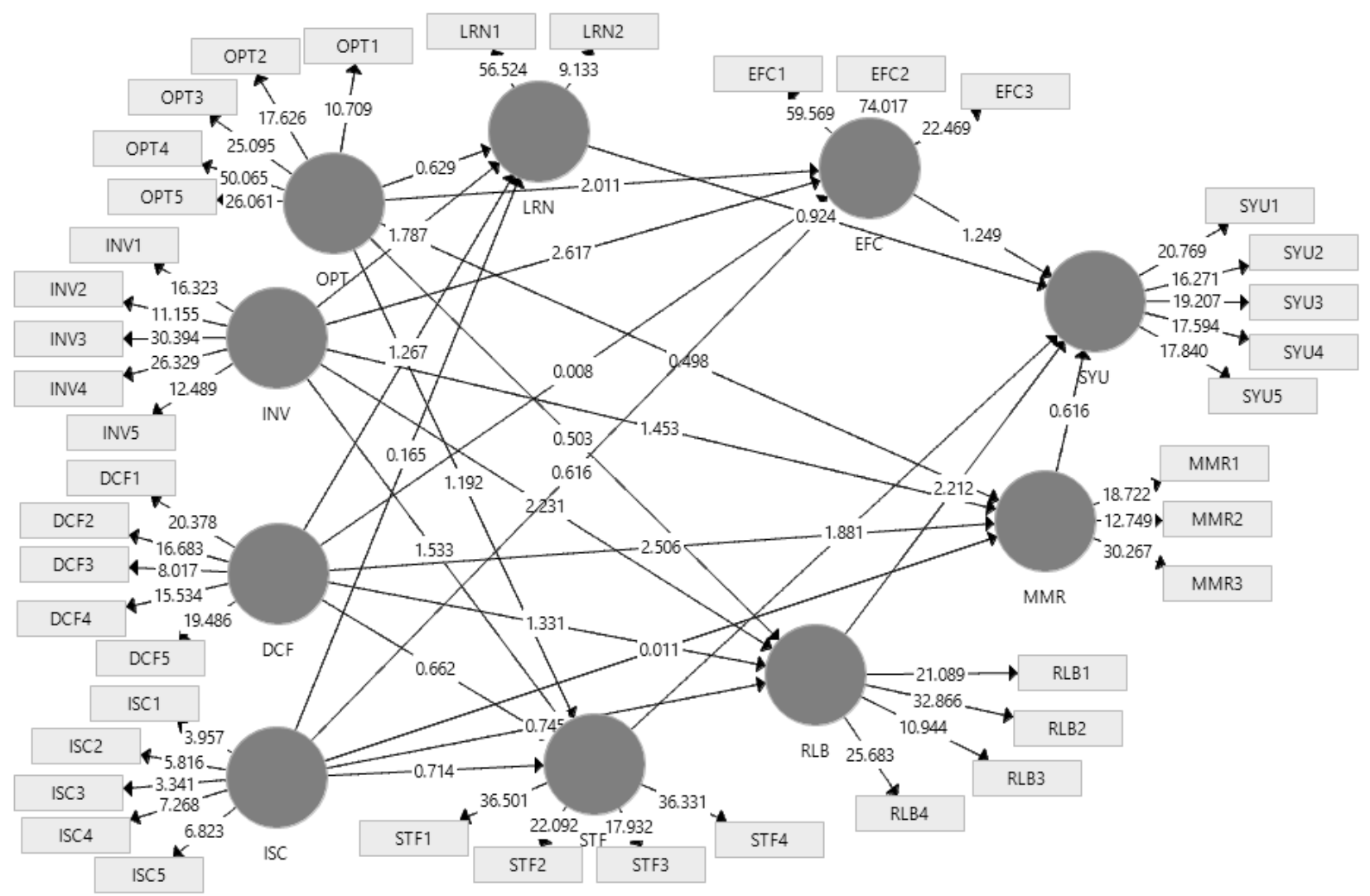

Figure 3. Research model TRU

Table 5. Construct reliability and validity

\begin{tabular}{ccccc}
\hline & $\begin{array}{c}\text { Cronbach's } \\
\text { Alpha }\end{array}$ & rho_A & $\begin{array}{c}\text { Composite } \\
\text { Reliability }\end{array}$ & $\begin{array}{c}\text { Average } \\
\text { Variance } \\
\text { Extracted } \\
\text { (AVE) }\end{array}$ \\
\hline DCF & 0.916 & 0.929 & 0.938 & 0.753 \\
EFC & 0.926 & 0.929 & 0.944 & 0.772 \\
INV & 0.885 & 0.890 & 0.916 & 0.687 \\
ISC & 0.885 & 0.895 & 0.915 & 0.684 \\
LRN & 0.847 & 0.904 & 0.882 & 0.603 \\
MMR & 0.882 & 0.885 & 0.919 & 0.740 \\
OPT & 0.717 & 0.895 & 0.867 & 0.766 \\
RLB & 0.949 & 0.964 & 0.967 & 0.908 \\
STF & 0.842 & 0.849 & 0.905 & 0.761 \\
SYU & 0.929 & 0.929 & 0.947 & 0.817 \\
\hline
\end{tabular}

As we can see from the table above, the composite 0,708 and have high levels of internal consistency reliability for all the reflective constructs are higher than reliability.

\begin{tabular}{|c|c|c|c|c|c|c|c|c|c|c|}
\hline \multicolumn{11}{|c|}{ Table 6. Outer loadings } \\
\hline & DCF & EFC & INV & ISC & LRN & MMR & OPT & RLB & STF & SYU \\
\hline DCF1 & 0.857 & & & & & & & & & \\
\hline DCF2 & 0.790 & & & & & & & & & \\
\hline DCF3 & 0.774 & & & & & & & & & \\
\hline DCF4 & 0.841 & & & & & & & & & \\
\hline DCF5 & 0.870 & & & & & & & & & \\
\hline $\mathrm{EFC1}$ & & 0.958 & & & & & & & & \\
\hline $\mathrm{EFC} 2$ & & 0.970 & & & & & & & & \\
\hline $\mathrm{EFC} 3$ & & 0.930 & & & & & & & & \\
\hline INV1 & & & 0.805 & & & & & & & \\
\hline INV2 & & & 0.758 & & & & & & & \\
\hline INV3 & & & 0.908 & & & & & & & \\
\hline INV4 & & & 0.865 & & & & & & & \\
\hline INV5 & & & 0.800 & & & & & & & \\
\hline ISC 1 & & & & 0.684 & & & & & & \\
\hline ISC2 & & & & 0.789 & & & & & & \\
\hline ISC3 & & & & 0.645 & & & & & & \\
\hline
\end{tabular}

Assessment of Readiness and Usability of Information Systems Use

(Dwi Yuniarto, A'ang Subiyakto, Aedah Binti Abd. Rohman, Reny Rian Marliana) 


\begin{tabular}{|c|c|c|c|c|c|c|c|c|c|c|}
\hline & DCF & EFC & INV & ISC & LRN & MMR & OPT & RLB & STF & SYU \\
\hline ISC4 & & & & 0.874 & & & & & & \\
\hline ISC5 & & & & 0.865 & & & & & & \\
\hline LRN1 & & & & & 0.944 & & & & & \\
\hline LRN2 & & & & & 0.801 & & & & & \\
\hline MMR1 & & & & & & 0.853 & & & & \\
\hline MMR2 & & & & & & 0.845 & & & & \\
\hline MMR3 & & & & & & 0.917 & & & & \\
\hline OPT1 & & & & & & & 0.733 & & & \\
\hline OPT2 & & & & & & & 0.840 & & & \\
\hline OPT3 & & & & & & & 0.906 & & & \\
\hline OPT4 & & & & & & & 0.940 & & & \\
\hline OPT5 & & & & & & & 0.904 & & & \\
\hline RLB1 & & & & & & & & 0.878 & & \\
\hline RLB2 & & & & & & & & 0.899 & & \\
\hline RLB3 & & & & & & & & 0.806 & & \\
\hline RLB4 & & & & & & & & 0.854 & & \\
\hline STF1 & & & & & & & & & 0.928 & \\
\hline STF2 & & & & & & & & & 0.888 & \\
\hline STF3 & & & & & & & & & 0.874 & \\
\hline STF4 & & & & & & & & & 0.925 & \\
\hline SYU1 & & & & & & & & & & 0.895 \\
\hline SYU2 & & & & & & & & & & 0.885 \\
\hline SYU3 & & & & & & & & & & 0.906 \\
\hline SYU4 & & & & & & & & & & 0.853 \\
\hline SYU5 & & & & & & & & & & 0.854 \\
\hline
\end{tabular}

From the Table 6, we can see that the outer loadings of indicator ISC1 and ISC3 are below to 0.7, these two indicators need to be analyzed of the impact of indicator deletion on AVE and composite reliability (see Table 5). If the deletion increasing those measurements then the reflective indicators need to be removed from the model, but if the deletion does not increase those measurements the reflective indicators need to be retained.

Table 7. Composite reliability from all models

\begin{tabular}{lcc}
\hline $\begin{array}{l}\text { Composite } \\
\text { Reliability }\end{array}$ & $\begin{array}{c}\text { Full } \\
\text { Model }\end{array}$ & $\begin{array}{c}\text { Model 2 (Deletion of } \\
\text { Indicators ISC1 and } \\
\text { ISC3) }\end{array}$ \\
\hline DCF & 0.938 & 0.915 \\
EFC & 0.944 & 0.967 \\
INV & 0.916 & 0.916 \\
ISC & 0.915 & 0.894 \\
LRN & 0.882 & 0.867 \\
MMR & 0.919 & 0.905 \\
OPT & 0.867 & 0.938 \\
RLB & 0.967 & 0.919 \\
STF & 0.905 & 0.947 \\
SYU & 0.947 & 0.944 \\
\hline
\end{tabular}

From table 7, we get that deletion of indicators ISC1 and ISC3 has increased the composite reliability.

The AVE for all models are higher than 0,5 , so the convergent validity confirmed. From table 8 we also get that all the indicator's outer loadings on a construct are higher than its cross-loadings with other constructs, then the discriminant validity confirmed [39].
Table 8. Convergent validity from all models

\begin{tabular}{lcc}
\hline AVE & $\begin{array}{c}\text { Full } \\
\text { Model }\end{array}$ & $\begin{array}{c}\text { Model 2 (Deletion } \\
\text { of Indicators ISC1 } \\
\text { and ISC3) }\end{array}$ \\
\hline DCF & 0.753 & 0.684 \\
EFC & 0.772 & 0.908 \\
INV & 0.687 & 0.687 \\
ISC & 0.684 & 0.737 \\
LRN & 0.603 & 0.766 \\
MMR & 0.740 & 0.761 \\
OPT & 0.766 & 0.753 \\
RLB & 0.908 & 0.740 \\
STF & 0.761 & 0.817 \\
SYU & 0.817 & 0.772 \\
\hline
\end{tabular}

The determination of whether or not the hypothesis is based on the research model we can see at Tabel 9. To assess the significance of path coefficients we use significant level $5 \%$ and one-tailed test. The significance level is 1,64 .

$\mathrm{R}^{2}$ values of endogen constructs System Usability and Memorability are respectively substantial, meanwhile the endogen constructs Efficiency, Learnability are respectively weak and endogen construct Reliability, Satisfaction is respectively moderate (Tabel 10). Meanwhile, from Tabel 11, we can see all the exogenous construct's contribution to its endogenous latent variable [39].

Table 9. Cross loadings of model 2

\begin{tabular}{lcccccccccc}
\hline & DCF & EFC & INV & ISC & LRN & MMR & OPT & RLB & STF & SYU \\
\hline DCF1 & 0.857 & -0.341 & -0.379 & 0.671 & -0.353 & -0.471 & -0.398 & -0.412 & -0.420 & -0.339 \\
DCF2 & 0.790 & -0.206 & -0.367 & 0.594 & -0.495 & -0.483 & -0.317 & -0.441 & -0.450 & -0.337 \\
DCF3 & 0.774 & -0.329 & -0.349 & 0.598 & -0.305 & -0.305 & -0.288 & -0.224 & -0.191 & -0.065 \\
DCF4 & 0.841 & -0.348 & -0.354 & 0.600 & -0.315 & -0.493 & -0.421 & -0.432 & -0.269 & -0.226 \\
DCF5 & 0.870 & -0.243 & -0.272 & 0.658 & -0.271 & -0.456 & -0.349 & -0.393 & -0.310 & -0.170 \\
EFC1 & -0.314 & 0.958 & 0.561 & -0.335 & 0.448 & 0.527 & 0.493 & 0.534 & 0.642 & 0.664 \\
EFC2 & -0.292 & 0.970 & 0.511 & -0.268 & 0.379 & 0.547 & 0.490 & 0.523 & 0.650 & 0.590 \\
EFC3 & -0.410 & 0.930 & 0.467 & -0.373 & 0.339 & 0.528 & 0.517 & 0.437 & 0.570 & 0.421 \\
INV1 & -0.474 & 0.532 & 0.805 & -0.336 & 0.289 & 0.453 & 0.490 & 0.423 & 0.385 & 0.451 \\
INV2 & -0.283 & 0.507 & 0.758 & -0.277 & 0.361 & 0.336 & 0.651 & 0.234 & 0.372 & 0.376 \\
INV3 & -.0358 & 0.475 & 0.908 & -0.196 & 0.418 & 0.292 & 0.532 & 0.287 & 0.339 & 0.383 \\
\hline
\end{tabular}




\begin{tabular}{|c|c|c|c|c|c|c|c|c|c|c|}
\hline & DCF & EFC & INV & ISC & LRN & MMR & OPT & RLB & STF & SYU \\
\hline INV4 & -0.328 & 0.407 & 0.865 & -0.181 & 0.412 & 0.324 & 0.479 & 0.379 & 0.349 & 0.589 \\
\hline INV5 & -0.256 & 0.281 & 0.800 & -0.087 & 0.378 & 0.301 & 0.407 & 0.267 & 0.294 & 0.334 \\
\hline ISC2 & 0.639 & -0.277 & -0.282 & 0.813 & -0.244 & -0.371 & -0.195 & -0.339 & -0.318 & -0.213 \\
\hline ISC4 & 0.741 & -0.116 & -0.213 & 0.868 & -0.181 & -0.325 & -0.286 & -0.261 & -0.185 & -0.109 \\
\hline ISC 5 & 0.604 & -0.404 & -0.202 & 0.893 & -0.384 & -0.369 & -0.373 & -0.374 & -0.390 & -0.334 \\
\hline LRN1 & -0.421 & 0.418 & 0.450 & -0.356 & 0.944 & 0.478 & 0.417 & 0.504 & 0.520 & 0.550 \\
\hline LRN2 & -0.314 & 0.277 & 0.306 & -0.195 & 0.801 & 0.311 & 0.228 & 0.224 & 0.240 & 0.234 \\
\hline MMR1 & -0.485 & 0.493 & 0.328 & -0.413 & 0.617 & 0.853 & 0.294 & 0.707 & 0.692 & 0.495 \\
\hline MMR2 & -0.420 & 0.480 & 0.347 & -0.303 & 0.273 & 0.845 & 0.273 & 0.595 & 0.702 & 0.539 \\
\hline MMR3 & -0.516 & 0.494 & 0.412 & -0.377 & 0.346 & 0.917 & 0.416 & 0.616 & 0.719 & 0.561 \\
\hline OPT1 & -0.381 & 0.411 & 0.418 & -0.334 & 0.261 & 0.288 & 0.733 & 0.206 & 0.214 & 0.190 \\
\hline OPT2 & -0.366 & 0.461 & 0.603 & -0.243 & 0.340 & 0.245 & 0.840 & 0.234 & 0.329 & 0.350 \\
\hline OPT3 & -0.309 & 0.476 & 0.573 & -0.278 & 0.284 & 0.310 & 0.906 & 0.208 & 0.376 & 0.426 \\
\hline OPT4 & -0.370 & 0.531 & 0.576 & -0.363 & 0.358 & 0.363 & 0.940 & 0.250 & 0.427 & 0.466 \\
\hline OPT5 & -0.453 & 0.392 & 0.521 & -0.257 & 0.436 & 0.420 & 0.904 & 0.277 & 0.402 & 0.402 \\
\hline RLB1 & -.0394 & 0.357 & 0.263 & -0.293 & 0.389 & 0.587 & 0.103 & 0.878 & 0.612 & 0.561 \\
\hline RLB2 & -0.391 & 0.468 & 0.388 & -0.304 & 0.319 & 0.594 & 0.295 & 0.898 & 0.642 & 0.685 \\
\hline RLB3 & -0.453 & 0.512 & 0.393 & -0.399 & 0.481 & 0.688 & 0.267 & 0.807 & 0.647 & 0.557 \\
\hline RLB4 & -0.388 & 0.465 & 0.289 & -0.343 & 0.374 & 0.643 & 0.256 & 0.854 & 0.695 & 0.657 \\
\hline STF1 & -0.392 & 0.499 & 0.328 & -0.335 & 0.482 & 0.755 & 0.349 & 0.701 & 0.928 & 0.616 \\
\hline STF2 & -0.373 & 0.743 & 0.418 & -0.400 & 0.432 & 0.677 & 0.451 & 0.622 & 0.888 & 0.730 \\
\hline STF3 & -0.308 & 0.467 & 0.362 & -0.238 & 0.400 & 0.723 & 0.334 & 0.714 & 0.874 & 0.653 \\
\hline STF4 & -0.410 & 0.623 & 0.413 & -0.344 & 0.391 & 0.766 & 0.338 & 0.702 & 0.925 & 0.710 \\
\hline SYU1 & -0.332 & 0.525 & 0.524 & -0.250 & 0.481 & 0.561 & 0.383 & 0.586 & 0.660 & 0.895 \\
\hline SYU2 & -0.251 & 0.459 & 0.476 & -0.269 & 0.479 & 0.518 & 0.374 & 0.632 & 0.662 & 0.885 \\
\hline SYU3 & -0.146 & 0.409 & 0.414 & -0.162 & 0.373 & 0.466 & 0.328 & 0.562 & 0.561 & 0.906 \\
\hline SYU4 & -0.237 & 0.545 & 0.371 & -0.252 & 0.443 & 0.519 & 0.322 & 0.752 & 0.681 & 0.853 \\
\hline SYU5 & -0.306 & 0.653 & 0.503 & -0.271 & 0.367 & 0.602 & 0.484 & 0.595 & 0.721 & 0.854 \\
\hline
\end{tabular}

Table 10. Assessment of the significance of path coefficients

\begin{tabular}{|c|c|c|c|c|c|c|}
\hline & $\begin{array}{c}\text { Original } \\
\text { Sample (O) }\end{array}$ & $\begin{array}{c}\text { Sample } \\
\text { Mean (M) } \\
\end{array}$ & $\begin{array}{c}\text { Standard Deviation } \\
\text { (STDEV) }\end{array}$ & $\begin{array}{c}\text { T Statistics } \\
(\mid \text { O/STDEV } \mid)\end{array}$ & P Values & Results \\
\hline DCF -> EFC & 0.063 & 0.059 & 0.224 & 0.281 & 0.779 & Not Significant \\
\hline DCF $->$ LRN & -0.232 & -0.230 & 0.188 & 1.237 & 0.216 & Not Significant \\
\hline $\begin{array}{c}\text { DCF -> } \\
\text { MMR }\end{array}$ & -0.407 & -0.401 & 0.165 & 2.471 & 0.014 & Significant \\
\hline DCF $->$ RLB & -0.305 & -0.307 & 0.198 & 1.538 & 0.125 & Not Significant \\
\hline DCF -> STF & -0.131 & -0.141 & 0.187 & 0.702 & 0.483 & Not Significant \\
\hline DCF $->$ SYU & -0.123 & -0.120 & 0.168 & 0.730 & 0.466 & Not Significant \\
\hline $\mathrm{EFC}->\mathrm{SYU}$ & 0.147 & 0.153 & 0.122 & 1.201 & 0.230 & Not Significant \\
\hline INV $->$ EFC & 0.349 & 0.365 & 0.134 & 2.603 & 0.010 & Significant \\
\hline INV -> LRN & 0.269 & 0.259 & 0.152 & 1.769 & 0.077 & Significant \\
\hline $\begin{array}{l}\text { INV -> } \\
\text { MMR }\end{array}$ & 0.194 & 0.200 & 0.129 & 1.497 & 0.135 & Not Significant \\
\hline INV -> RLB & 0.275 & 0.270 & 0.136 & 2.021 & 0.044 & Significant \\
\hline INV $->$ STF & 0.228 & 0.231 & 0.150 & 1.518 & 0.130 & Not Significant \\
\hline INV -> SYU & 0.257 & 0.281 & 0.118 & 2.169 & 0.031 & Significant \\
\hline ISC -> EFC & -0.230 & -0.207 & 0.193 & 1.053 & 0.293 & Not Significant \\
\hline ISC -> LRN & -0.051 & -0.068 & 0.190 & 0.266 & 0.790 & Not Significant \\
\hline ISC -> MMR & -0.034 & -0.033 & 0.188 & 0.182 & 0.856 & Not Significant \\
\hline ISC $->$ RLB & -0.108 & -0.110 & 0.185 & 0.582 & 0.561 & Not Significant \\
\hline ISC $->$ STF & -0.154 & -0.162 & 0.193 & 0.796 & 0.426 & Not Significant \\
\hline ISC -> SYU & -0.140 & -0.147 & 0.144 & 0.971 & 0.332 & Not Significant \\
\hline LRN $->$ SYU & 0.129 & 0.109 & 0.144 & 0.895 & 0.371 & Not Significant \\
\hline $\begin{array}{c}\text { MMR -> } \\
\text { SYU }\end{array}$ & -0.163 & -0.094 & 0.263 & 0.618 & 0.537 & Not Significant \\
\hline OPT $->$ EFC & 0.265 & 0.246 & 0.131 & 2.021 & 0.044 & Significant \\
\hline OPT $->$ LRN & 0.107 & 0.115 & 0.179 & 0.595 & 0.552 & Not Significant \\
\hline $\begin{array}{c}\text { OPT -> } \\
\text { MMR }\end{array}$ & 0.071 & 0.087 & 0.153 & 0.461 & 0.645 & Not Significant \\
\hline OPT $->$ RLB & -0.067 & -0.059 & 0.135 & 0.497 & 0.619 & Not Significant \\
\hline OPT -> STF & 0.160 & 0.153 & 0.142 & 1.124 & 0.262 & Not Significant \\
\hline OPT $->$ SYU & 0.091 & 0.085 & 0.118 & 0.772 & 0.441 & Not Significant \\
\hline RLB $->$ SYU & 0.354 & 0.370 & 0.154 & 2.295 & 0.022 & Significant \\
\hline STF -> SYU & 0.461 & 0.388 & 0.247 & 1.865 & 0.063 & Significant \\
\hline
\end{tabular}


Table 11. R Square

\begin{tabular}{ccc}
\hline & R Square & R Square Adjusted \\
\hline EFC & 0.374 & 0.329 \\
LRN & 0.278 & 0.226 \\
MMR & 0.344 & 0.296 \\
RLB & 0.275 & 0.222 \\
STF & 0.273 & 0.220 \\
SYU & 0.652 & 0.620 \\
\hline
\end{tabular}

Table 12. F- Square

\begin{tabular}{|c|c|c|c|c|c|c|c|c|c|c|}
\hline & DCF & EFC & INV & ISC & LRN & MMR & OPT & RLB & STF & SYU \\
\hline DCF & & 0.002 & & & 0.028 & 0.095 & & 0.048 & 0.009 & \\
\hline EFC & & & & & & & & & & 0.035 \\
\hline INV & & 0.113 & & & 0.058 & 0.033 & & 0.060 & 0.041 & \\
\hline ISC & & 0.028 & & & 0.002 & 0.001 & & 0.007 & 0.014 & \\
\hline LRN & & & & & & & & & & 0.035 \\
\hline MMR & & & & & & & & & & 0.024 \\
\hline OPT & & 0.064 & & & 0.009 & 0.004 & & 0.004 & 0.020 & \\
\hline RLB & & & & & & & & & & 0.137 \\
\hline STF & & & & & & & & & & 0.153 \\
\hline SYU & & & & & & & & & & \\
\hline
\end{tabular}

\section{CONCLUSION}

In accordance with the purpose of the study, evaluation of questionnaires using statistical analysis through validity and reliability used as material for revision of the model and questionnaires that have previously been built through the stages of integration and adoption of several models. The results of this study there are no results from the evaluation that researchers must do to change models and questionnaires, only for researchers who are interested in research in the area of research regarding the use of information systems, it becomes its own attraction to further develop and measure further.

It needs to be a very big concern regarding the sample used in this study, considering the sample used only in private universities in Indonesia that have solid activities in the use of information systems, it is better for other researchers to try to apply the measurement model that has been built including the questionnaire in the different.

\section{REFERENCES}

[1] M.-C. Wu, "A Study on the Willingness to Use Information System of Sport Event Based on Information System Success Model," The Journal of Human Resource and Adult Learning, Vol. 9, Num. 2, December 2013 issue, 2013.

[2] D. Yuniarto, A. Subiyakto, E. Firmansyah, D. Herdiana, M. Suryadi, and A. A. Rahman, "Integrating the Readiness and Usability Models for Assessing the Information System Use," The 6th International Conference on Cyber and IT Service Management (CITSM 2018) Medan, August 7-9, 2018, 2018.

[3] A. Subiyakto, "Development of the Readiness and Success Model for Assessing the Information System Integration," pp. 1-10, 2017.

[4] A. Subiyakto, R. Rosalina, M. C. Utami, N. Kumaladewi, and S. J. Putra, "The Psychometric and Interpretative Analyses for Assessing the End-User Computing Satisfaction Questionnaire," in 5th International Conference on Information Technology for Cyber and IT Service Management (CITSM) 2017 Denpasar, Bali, 2017: IEEE.

[5] A. Subiyakto, D. Septiandani, E. Nurmiati, Y. Durachman, M. Kartiwi, and A. R. Ahlan, "Managers Perceptions towards the Success of E-Performance Reporting System,"
TELKOMNIKA (Telecommunication Computing Electronics and Control), vol. 15, no. 3, pp. 1389-1396, 2017.

[6] A. Subiyakto, "Development of The Readiness and Success Model for Assessing the Information System Integration," presented at the International Conference on Science and Technology (ICOSAT) 2017, Jakarta, 08 October 2017 2017.

[7] S. J. Putra, A. Subiyakto, A. R. Ahlan, and M. Kartiwi, "A Coherent Framework for Understanding the Success of an Information System Project," TELKOMNIKA (Telecommunication Computing Electronics and Control), vol. 14, no. 1, pp. 302-302, 2016.

[8] A. Subiyakto, A. R. Ahlan, M. Kartiwi, and S. J. Putra, "Measurement of the information system project success of the higher education institutions in Indonesia: a pilot study," International Journal of Business Information System, vol. 23, no. 2, pp. 229-247, 2016.

[9] A. Subiyakto, A. R. Ahlan, M. Kartiwi, S. J. Putra, and Y. Durachman, "The User Satisfaction Perspectives of the Information System Projects," Indonesian Journal of Electrical Engineering and Computer Science, vol. 4, no. 1, 2016.

[10] A. Subiyakto, A. R. Ahlan, M. Kartiwi, and H. T. Sukmana, "Influences of the Input Factors towards Success of An Information System Project," TELKOMNIKA (Telecommunication Computing Electronics and Control), vol. 13, no. 2, p. 686, 2015.

[11] A. Subiyakto, A. R. Ahlan, S. J. Putra, and M. Kartiwi, "Validation of Information System Project Success Model," SAGE Open, vol. 5, no. 2, p. 215824401558165, 2015.

[12] A. Subiyakto, A. R. Ahlan, M. Kartiwi, and H. T. Sukmana, "Measurement of information system project success based on perceptions of the internal stakeholders," International Journal of Electrical and Computer Engineering, vol. 5, no. 2, pp. 271-279, 2015.

[13] A. Subiyakto and A. R. Ahlan, "Implementation of InputProcess-Output Model for Measuring Information System Project Success," TELKOMNIKA Indonesian Journal of Electrical Engineering, vol. 12, no. 7, 2014.

[14] A. Subiyakto, A. R. Ahlan, and H. T. Sukmana, "An Alternative Method for Determining Critical Success Factors of Information System Project," TELKOMNIKA Telecommunication, Computing, Electronics and Control, vol. 12, no. 3, pp. 665-674, 20142014. 
[15] A. Subiyakto and A. R. Bin Ahlan, "A coherent framework for understanding critical success factors of ICT project environment," International Conference on Research and Innovation in Information Systems, ICRIIS, vol. 2013, pp. 342-347, 2013.

[16] R. B. CRUZ, "Land Use Information System for Local Government: The Case of Naga City, Philippines," SYSTEMICS, CYBERNETICS AND INFORMATICS ISSN: 1690-4524, vol. ISSN: 1690-4524 SYSTEMICS, CYBERNETICS AND INFORMATICS no. VOLUME 4 NUMBER 6.

[17] D. Yuniarto, "Analisis Penerimaan Penggunaan Aplikasi Laporan BKD dan Evaluasi Pelaksanaan Tridharma PT Online menggunakan TAM," 2018.

[18] D. Yuniarto, "Analisis Penerimaan Grab di Kabupaten Sumedang," vol. 5, pp. 0-4, 2017.

[19] G. Gusti, "Pengukuran Pengaruh Kesiapan Terhadap Keberhasilan Penerapan Sistem Ubiquitous Computing di UIN Syarif," no. February, 2018.

[20] M. Kartiwi, "Model Keberhasilan Proyek Sistem Informasi : Penjelasan Ringkas Pencatatan Hak Cipta No . Universitas Islam Negeri ( UIN ) Syarif Hidayatullah Jakarta," no. 01835, 2017.

[21] H. A. Qadrya, "Faktor-faktor yang Mempengaruhi Kesiapan Penerapan Sistem Single Sign On di UIN Syarif Hidayatullah Jakarta," no. January, 2018.

[22] F. A. Uin, "SISTEM SINGLE SIGN ON DI UIN SYARIF," no. February, 2018.

[23] H. Jeskanen-Sundstrom, "ICT Statistics at the New Millennium - Developing Official Statistics - Measuring the Diffusion of ICT and its Impacts," IAOS Satellite Meeting on Statistics for the Information Society August 30 and 31, 2001, Tokyo, Japan, 2001.

[24] R. D. Tobias, "An Introduction to Partial Least Squares Regression," SAS Institute Inc., Cary, NC.

[25] T. He and C. Zhu, "Digital informal learning among Chinese university students: the effects of digital competence and personal factors," International Journal of Educational Technology in Higher Education, vol. 14, no. $1,2017$.

[26] J. Henseler, G. Hubona, and P. A. Ray, "Using PLS path modeling in new technology research: updated guidelines," Industrial Management \& Data Systems, vol. 116, no. 1, pp. 2-20, 2016.

[27] L. A. Joia, L. F. D. Gutman, and V. Moreno, "Intention of use of home broker systems from the stock market investors' perspective," Journal of High Technology Management Research, vol. 27, no. 2, pp. 184-195, 2016.

[28] J. H. Joo, "Understanding Korean College Students' Social Commerce Behavior through an Integrated Model of Technology Readiness, Technology Acceptance Model, and Theory of Planned Behavior," Journal of Digital Convergence, vol. 13, no. 7, pp. 99-107, 2015.

[29] J. Luftman, K. Lyytinen, and T. B. Zvi, "Enhancing the Measurement of Information Technology (IT) Business Alignment and Its Influence on Company Performance," Journal of Information Technology, vol. 32, no. 1, pp. 2646, 2017.

[30] A. A. Rabaai, "An Empirical Investigation on the Adoption of e-Government in Developing Countries: The Case of Jordan," Computer and Information Science, vol. 8, no. 3, 2015.

[31] M. Sarstedt, C. M. Ringle, and J. F. Hair, "Partial Least Squares Structural Equation Modeling," Handbook of Market Research, no. May 2017, pp. 1-40, 2017.

[32] A. Subiyakto, A. R. Ahlan, M. Kartiwi, and H. T. Sukmana, "Influences of the Input Factors towards Success of An Information System Project," TELKOMNIKA (Telecommunication Computing Electronics and Control), vol. 13, no. 2, pp. 686-686, 2015.

[33] A. Subiyakto, R. Rosalina, M. C. Utami, N. Kumaladewi, and S. J. Putra, "The psychometric and interpretative analyses for assessing the end-user computing satisfaction questionnaire," 2017 5th International Conference on Cyber and IT Service Management, CITSM 2017, no. November, 2017.

[34] P. Seminar et al., "Algoritma Enkripsi Citra," vol. 4, no. November, pp. 117-126, 2014.

[35] W. M. A. B. W. Afthanorhan, "A Comparison Of Partial Least Square Structural Equation Modeling (PLS-SEM) and Covariance Based Structural Equation Modeling (CBSEM) for Confirmatory Factor Analysis," International Journal of Engineering Science and Innovative Technology (IJESIT) Volume 2, Issue 5, September 2013, 2013.

[36] Z. Awang, A. Afthanorhan, and M. Mamat, "The Likert scale analysis using parametric based Structural Equation Modeling ( SEM )," Computational Methods in Social Sciences, vol. 4, no. 1, pp. 13-21, 2015.

[37] A. Parasuraman and C. L. Colby, "An Updated and Streamlined Technology Readiness Index: TRI 2.0," Journal of Service Research, vol. 18, no. 1, pp. 59-74, 2015.

[38] J. Nielsen, "Usability 101 : Introduction to Usability Why Usability is Important How to Improve Usability," Focus, pp. 1-4, 2006.

[39] J. F. Hair Jr, G. T. M. Hult, C. Ringle, and M. Sarstedt, $A$ primer on partial least squares structural equation modeling (PLS-SEM). Sage Publications, 2016. 